Again, it is very uncommon to meet with an instance of acute necrosis of the head of the femur-though I have known the occurrence of such ar affection -and this is as one would expect from the position and connections of this part of the bone; while, during the last two years, fifty cases have come to this out-patients' room, of hip-joint disease, where there was evidence of a carious condition of the head of the bone. On the other hand, the shaft and lower end of the femur are commonly enough attacked by necrosis. Again, I would remind you of the extreme infrequency of necrosis of the bones of the tarsus and carpus; of their liability to caries; of the liability of the phalanges of both fingers and toes to necrosis; of their general exemption from caries.

This difference in the liability of bones to be attacked by one of the two diseases, necrosis or caries, seems to depend (I repeat) -

1. On the composition of the bone, whether the cancellous or compact structure predominate ;

2 . On the position of the bone, whether it be protected from external violence and changes of temperature, or not;

3 . On the presence and extent of, or the absence of, a fibrous periosteal investment.

To both necrosis and caries, the bones of children and growing adults are particularly liable ; and I believe this liability is due to the increased vascularity and sensibility of these parts during their period of growth and development; rendering them highly resentful of external injuries, and prone to serious disturbances in their circulation, when exposed to extremes or sudden variations in the external temperature.

If any one will take the trouble to examine the membrane covering one of the long bones of a child, in its soft, vascular, and almost succulent texture, and in the ease with which it is separable from the bone, he will scarcely recognise its identity with the dense, dry, and tightly clinging, fibrous periosteum of a full-grown adult, from which it differs in texture almost as much as does a mucous from a serous membrane. During the last years of growth, and the early period of manhood, when growth has ceased, the soft, vascular, and rapidly ossifying structure forming the deepest layer of the periosteum, disappears; the membrane itself becomes thinner, from ossification of its deeper layers ; it is denser, and more tightly stretched over the subjacent bone, with which its vascular connections are now less intimate, the blood-vessels having diminished both in number and size.

It is to this vascularity of the deeper periosteal layer in young persons that I would direct your attention, since it is this that peculiarly predisposes them to subperiosteal effusions from slight external causes-a condition, as I hope to show you, always imperilling the vitality of the bone, and not unattended with danger to the individual.

$$
\text { [ } T \text { o be continued.] }
$$

A Capital Opening for a Physician. At 2 p.M. this morning, a noted physician of Carlisle reached Harrisburgh, Pennsylvania, in a bugg, having left that place iwo hours previously under the impression that the rebels were within one mile of him. He abandoned his sonse, furniture, and property of all description. (Tele(r) $(n+\cdots)$

\section{Clinical allectures}

DEIIVERED AT

\section{CHARING CROSS HOSPITAL.}

IY

HYDE SALTER, M.D., F.R.S.,

FELLOW OF THE ROYAL COLLEGE OF PHYSICIANS; LECTURER ON PHYSIOLOGY AND PATHOLOGY AT CHARING CROSS HOSPITAL MEDICAL SCHOOL; AND ASSISTANT. PHYSICIAN TO THE HOSPITAL.

Lecture VII.-ON Pleurisy. (Concluded.)

Case rr. Case IIr. The Pain of Pleurisy. The Diagnostic Bearings of Pain in the Side. The Nature of Pleuritic Pain; its Non-dependence on Friction; its Non-coincidence with the Inflammation; is probably Reflex. The Diagnosis of Pulmonary from Costal Pleurisy. Important Part which the Inflammatory Results play. The Pathology of Catarrhal Pleurisy. Prognosis. The Persistence of Pleuritic Friction. What determines the divers Results of Pleurisy? Treatment.

Case II. Sarah Russell, aged 19, presented herself among the out-patients at Charing Cross Hospital, May 2nd, complaining of severe stitch in her side, just under the right breast. It is much aggravated by attempting to take a deep breath; indeed, the pain is so great on trying to make a full inspiration that it is almost impossible to do so. She cannot lie on her right side. She dates the pain from a severe cold she caught on the night of the illuminations celebrating the Prince of Wales's marriage. This cold was attended with shivering, pains in the limbs, feverishness, and cough. A few days later the pain in the side was superadded, and has not left her since. She has now had it for three weeks.

On examining the chest, a strong pleuritic friction was heard at the seat of the pain, at the termination both of inspiration and expiration, especially the former. In every other respect the sounds of the chest were natural. There was no fever; the patient was pale and weak, with a depressed and rather anxious expression. I ordered perfect rest, the infriction of a sedative liniment, and internally opium, chloric ether, and quinine. $I$ advised this patient to come into the hospital, but she refused.

A week afterwards she presented herself again, and reported that the right side was quite well, but that the pain had moved to the left side. Bearing in mind the physical evidence of the organic nature of the cause of the pain, I felt rather sceptical of this migration. On applying my stethoscope to the original seat of the suund and pain I found that all trace of rubbing was gone. But what was my surprise, on applying the stethoscope to the opposite side, to find an exactly similar friction-sound in an exactly corresponding situation : So that while the pleurisy, with its results, had been vanishing on one side it had been developing itself on the opposite. The two points, right and left, where the friction was heard, were exactly symmetrical; its disappearance on the right side was cornplete-not a trace was left; whilst on the left it was even louder than it had been on the right. The patient appeared to have caught no fresh cold; in her general condition she was better; and, had it not been for the pain, would have considered herself well, except that she was weak. She still refused to come into the hospital, and I prescribed the same treatment as I had done before. Since that time I have not seen her.

Case IIr. Thomas Wingall, aged 17, a tall, well-made youth, with very little aspect of illness about him, a but. 
cher by trade, and in bis occupation much exposed to the weather; temperate.

Three weeks ago he caught cold; had headache, and vomited, coughed, and spat. Five days after he was attacked with pain under his right scapula, passing along the axilla to under the right breast, and aggravated by taking a deep breath and coughing, but not by movements of the arm or body. He was obliged by the pain to lie on the opposite side, although the right side was that on which he was accustomed to lie. The pain was the most severe the first two days; since that it has gradually become less severe, especially in the day. It is worst in the morning after he wakes until after he is up; it then gets better throughout the day, and comes on again in the evening, particularly on going to bed. $\mathrm{He}$ went on in this way for a fortnight, and then came to me, May 15th, among the out-patients at the hospital.

On examining his chest, I immediately found loud rubbing-sound in the upper part of the right mammary region, the most intense above and to the inner side of the right nipple, but extending thence about two inches upwards and inwards over the inner extremities and cartilages of the second, third, and fourth ribs. Sound audible at the termination both of inspiration and expiration, especially inspiration; the intensity of the sound proportionate to the length and depth of the respiratory act. The fremitus at the termination of each inspiration (especially if deep) distinctly perceptible by the hand. He eats, drinks, and sleeps perfectly well, and has done so since the first day or two of the attack, and he has not laid by from his work for a single day. Pulse 80 ; respiration 18 . The treatment consisted of local sedative, combined with iodine, and internally of iodide of potassium, quinine, and iron. As much rest as possible was enjoined.

May 20th. The pain is nearly gone ; what little there remains now is chiefly felt beneath the scapula. Friction as loud as eiver; its maximum intensity at the junction of the bone and cartilage of the third rib.

You will observe that in all three cases the one circumstance that brought the patient to the hospital was pain in the side ; and pain of a peculiar character-severe, circumscribed, stabbing, and greatly aggravated by inspiration. In Franklin's case, as we have seen, this pain was of the most violent kind, resembling the plunges of neuralgia more than anything else. Now such a pain as this almost always accompanies pleurisy; it is rare to find pleurisy without it; and hence when such a pain is present pleurisy is the thing one always thinks of and looks out for. But pain in the side may arise from fifty causes besides pleurisy ; and since some of these are very trifling, while pleurisy is often a grave affection, the diagnosis of lateral pain frequently becomes a very momentous as well as interesting question. How then, in any given case, can we ascertain if pain in the side is due to pleurisy or not? I will endeavour to show you as clearly as I possibly can. But I must admit that the diagnosis is sometimes difficult.

If physical signs show the anatomical results of pleurisy to be present, then pleurisy clearly exists or has existed, and the pain in the side is probably due to it.

But supposing there are no physical signs of pleurisy, is the pain on that account non-pleuritic? Certainly not. I believe it perfectly possible for pleurisy to be present and yet not reveal itself by any physical signs whatever; either because the inflammation is not intense enough to give rise to anatomical changes sufficiently marked to reveal themselves by physical signs, or because the stage of the inflammation is too early and the time has not yet arrived for the development of those changes. In such a case as this how are we to determine whether the pain points to pleurisy or not?

If there are other signs of lung mischief such as is often associated with pleurisy-as, for example, pneumonia, or tubercle, or cavity - then the pain is probably pleuritic.

If pressure between the ribs produces the pain, while pressure on the ribs does not, if inspiration is the great aggravator of the pain, if there is cough, if there is fever and much constitutional disturbance, and if the pain is circumscribed and a little without or below the nipple, then it is probably pleuritic, although there may be an entire absence of all physical signs of lung-disease.

If the pain is very severe and the pulse is unaffected, the pain is certainly (I think I may say) not pleuritic.

If moderate pressure over a rib, as well as between the ribs, produces the pain, the pain is not pleuritic.

If the movement of certain muscles which could not affect the pleura produces the pain, especially if there are other evidences of rheumatism, the pain is not pleuritic. Only yesterday we had two cases in which the diagnosis turned upon this point; contraction of the latissimus dorsi, that could not possibly have been appreciated by the pleura, gave rise to the pain in both cases. The verdict was rheumatism.

With regard to the influence that the evidence of rheumatism has upon the diagnosis, it must be admitted that it cuts both ways. For while rheumatism is one of the commonest causes of lateral pain simulating pleurisy, it is also, in its acute and febrile form, a very common cause of pleurisy itself; so that while the evidence of its presence might sug. gest the non-pleuritic nature of the pain, on the other hand it would afford an explanation of, and in some cases almost constitute a presumptive proof of, its true pleuritic character.

Doubtless, the case the most difficult of solution is the alternative between pleurisy and rheumatism of the intercostals. I have more than once been puzzled with it myself, and I have seen other and able men puzzled with it. There is in both cases the same superficial breathing, the same "stab" on attempting to take a full inspiration, the same lateral deculitus on the unaffected side, the same tenderness in the intercostal spaces. The rules of diagnosis that I have just mentioned to you will generally solve the mystery, but the most careful scrutiny may leave the question undecided.

The nature of this pain is no doubt the same as that of all other inflammatory hyperæsthesias, and, like them, the principal thing that aggravates it is mechanical disturbance; hence the intolerance of pressure and of stretching, hence the superficial breathing and the decubitus on the sound side. I do not believe that the friction of the roughened surfaces has anything to do with the pain, for two reasons : first, because you may have, as shown in the case of Wingall, pleuritic rubbing continuing after the pain has ceased, and when nothing is felt by the patient beyond a sense of the friction; and secondly, because the pain may be severe where the existence of effusion in considerable quantity prevents the contact of the two pleural surfaces. It has been said 
that as soon as effusion takes place the pain ceases. This is not true. In the case of Franklin the pain was of the most severe kind that I think I have ever witnessed in any case of pleurisy, while the pleural cavity was full of fluid-so full as to preclude all possibility of friction.

Does the seat of the pain coincide with the seat of the inflammation? As far as always occurring on the same side goes, I think it does. But I doubt if it does further. Certainly the seat of pain does not coincide with the seat of the greatest friction-sound. Thus, in the case of the lad Wingall, the maximum friction was about the cartilage of the third rib, where there was no pain ; while the chief pain was at the inferior angle of the scapula, where there was no rubbing. Moreover, there is a suspicious constancy about the seat of pleuritic pain, which cannot be explained by a similar constancy in the seat of the inflammation. We know from post mortem evidence that all parts of the pleura are liable to inflammation, while the seat of pleuritic pain is not liable to equal variety. For the seat of pleurisy there is no rule; for the seat of pleuritic pain there is a tolerably well marked rule :-beneath the nipple, at the inferior angle of the scapula, on the acromion, and beneath the false ribs, are its characteristic situations. Moreover, some of these situations are beyond the limits of the pleura, as on the acromion, and in the interval between the last rib and the crest of the ilium, where the chief pain in Franklin's case was felt. Moreover, the seat of pain may be covered with the finger when post mortem appearances show that the implication of the pleura has been almost universal. From all these considerations, I am inclined to think that pleuritic pain, as far as its distribution is concerned, is chiefly reflex; and that the constancy of its situation represents some law of reflex distribution, analogous to that which makes bronchial pain sternal, and colic pain umbilical, whatever may be the exact seat of the source of irritation.

I have often asked myself the question, in cases of pleurisy, whether both costal and pulmonary pleuræ were affected, or only one, and which; and if there were any means of diagnosing this point. Is it possible to answer this question? I think, to a certain extent, it is. I think one surface may be affected without the other; and certainly both may be affected at once. I think, if there is pleuritic effusion, and pressure between the ribs at the seat of pain increases the pain, that the costal pleura is affected. I think, on the other hand, that if, under such circumstances, there is no pain on pressure, the costal pleura is not affected. I think that if pneumonia coexists with the signs of pleurisy the implication of the pulmonary pleura is certain. I think that in all cases of pleuritic rubbing, both surfaces are certainly affected; perhaps one primarily, but both ultimately. In cases of pleuro-pneumonia in which there is friction-sound, the pulmonary pleura is probably affected first; and when that has been roughened, a similar state on the opposite surface of the costal pleura is set up by the chafing produced by the already roughened lung-surface. In pleuritic rubbing produced by traumatic injury of the thoracic parietes, the same events probably take place in a reversed order.

Observe the important part which the nature of the anatomical result of the inflammation plays in these cases. In the cases of Russell and Wingall, when the febrile stage of the cold passed off nothing remained but the inconvenience and annoyance of the stitch in the side. But Franklin's catarrhal attack left him not only with the pain, but with one lung. instead of two, with the incubus of a pleura-full of fluid upon his mediastinum and heart, and with all the circulatory and respiratory derangement and distress that must result from such a state of things. We see from this how, when hydrothorax is developed, it comes to constitute the substantive disease; -the pathology is lost, the morbid anatomy is everything.

What is the nature of the pleurisy in these cases? Some, no doubt, would say they are idiopathic. But I think, without adopting Serjeant Shee's definition of the word idiopathic,* I may show you that it would not be fair so to call them. It is quite clear that in all the cases the pleurisy was due to cold. Is this fact inconsistent with the general proposition with which I commenced my lecture-that serous inflammations preeminently point to states of bloodpoisoning? I think not. I think, on the contrary, that everything both in the etiology and clinical history of catarrh shows it to be a veritable toxhæmia. - a state of blood-contamination by a special materies morbi; and therefore pleurisy from catarrh is. but an example of a serous inflammation from a blood-poisoning. I do not see how it is possible to give any other reading to the phenomena of catarrh : -rigors, lassitude, headache, subjective pains in back and limbs, accelerated and enfeebled heart's action, loss of appetite, thirst-all the symptoms, in fact, of fevers of blood-origin; secondly, certain local inflammations of glandular or quasi-glandular parts; and thirdly, all this supervening on the suppression of the function of an eliminating surface which, taken in the aggregate, constitutes one of the largest glands of the body. And pleurisy is not the only serous inflammation that catarrh will produce; I am quite satisfied that I have seen, on three or four occasions, catarrhal peri- and endo-carditis. And, if you will not accuse me of reasoning in a circle, $I$ will say that, if I wanted a clinching and convincing proof of catarrhal fever being a true blood-poison state, I should find it in its tendency to give rise to serous inflammations.

The Prognosis in all these cases is entirely favourable. About the cases of the girl and lad, in which there was no effusion, there has been from first to last peculiarly little besides the local condition. That local condition has never been of a serious character. In the case of the girl, the pleura of the left side has merely to resume the state that the right pleura has already resumed, and which a few days has sufficed for it to resume, for her to be well. In the boy, as far as one can judge, the smoothing of the membrane will be a slower process. The man's case, as we have seen, is rapidly improving day by dayeach day the physical signs mark a subsidence in the effusion. It is now only a week and a day since I first listened to the chest and found, in the right half of it, apparently serum, et proterea nihil; and now there is but a small quantity in the most dependent part of the pleural cavity, and the lung has all but reoccupied its old situation. A few steps

* In the trial of Palmer for the murder of Cook, Serjeant Shee, in reply to a question from the bench as to what was the meaning of the constantly recurring word "idiopathice", said that it was a word em.
ployed by doctors to signify " that which was not understood". 
more in the same direction, and every drop of serum will be gone.

As far as physical signs go, I will venture to predict that the two last that will be lost will be the percussion-dulness and the diminished vocal fremitus ; the percussion-dulness last of all. It is wonderful how these two signs hang about after pleuritic effusion. Long after natural respiratory murmur has been reestablished, long after every trace of ægophony has disappeared, we shall probably find traces of these two signs. I venture to predict this on the strength of what I have observed in other cases. What is the cause of this lingering percussion-dulness and imperfectly re-established vocal fremitus I find it very difficult to guess. It is certainly not dependent on lung-compression or unabsorbed effusion. The only thing that I can imagine is, that the film of gelatinous fibrine-clot, to which I have already referred, so often found lining the cavity of the chest and covering the surface of lung in pleuritic hydrothorax, may, by intervening between the lung and the chest-wall, act as a damper, and in the one case stop the conduction of the percussion-stroke from without, and in the other the conduction of the bronchial voice-vibrations from within.

How soon the girl and the young man will lose their friction-sound it is impossible to say; nor does it matter. Pleuritic rubbing is one of the most variable and uncertain of physical signs as regards its duration, and one of the least important as regards its prognostic indications. You may just catch it for a day, or it may persist for weeks-yes, for months; and if it does so persist, a man is not a whit the worse for it, if he has recovered his health in every other respect. A man in whom all the functions of life are perfectly performed is not the less a sound man because you hear in his side a sound you do not hear in another's. I remember a patient in King's College Hospital, some years ago, who was kept in the hospital several weeks after he was in other respects well, in consequence of strong pleuritic sound and fremitus in his left pectoralis region. The man was a strong sturdy fellow, and made himself so generally useful that he became a sort of supernumerary servant of the hospital. We used to amuse ourselves, I remember, by making him strip, and feel with the palms of our hands on the pectoralis this friction-thrill each time he breathed. At last the physicians were ashamed of keeping him in any longer ; and he left the hospital with not only the sound, but the fremitus, as strong as ever. In Wingall's case, the friction will very likely last equally long. In the girl's case, however, I should expect that the rubbing would soon disappear, merely because it has so quickly disappeared on the opposite side.

There is one curious question that these cases suggest:-What is the determining cause that gives such different anatomical results in different cases of one and the same disease? Why in one of these cases should there have been effusion without roughening, and in the two others roughening without effu . sion? Shall we find the explanation of this difference in the condition of the blood? Shall we find it in any diathetic peculiarity? That it is not to be found in the nature of the exciting cause seems pretty evident, because in these three cases the exciting cause was one and the same.
I must not dismiss you without saying just one word about Treatment.

You will have observed that in all the cases my treatment was very simple. I aimed at three or four definite and intelligible objects with a view of placing the parts under the conditions most favourable for inducing and maintaining the natural reparative processes. These conditions in my opinion are:

To give functional and physical rest;

To allay pain by direct sedation ;

To keep up vital power.

To give functional and physical rest, because the part is crippled, and to work a crippled part is to keep it crippled; it is to keep it at a constant disadvantage; whereas it ought to be placed at an advantage, for something is to take place in it which is not taking place in the sound part, namely a reparative process over and above its ordinary nutrition and function.

To allay pain, because pain and healthy nutritional action are incompatible-as long as the one subsists the other will be suspended; and this, no doubt, from the baneful and disturbing influence which pain exercises on the nervous superintendance of the circulation of the part.

To keep up vital power, because the higher the standard at which this is kept, the more energetic will be the processes leading back to a condition of health, whether of deposition, or absorption, or whatever they may be.

In the cases of Russell and Wingall, in which there was no effusion, but merely the pain and friction, I endeavoured to secure these conditions by forbidding exertion, by the infriction of sedatives, and by the administration of quinine, etc. And even with the man Franklin I had but little more to do than this. I neither bled him, nor leeched him, nor blistered him, nor purged him, nor antimonialised him, nor mercurialised, nor "ised" him in any other way, except did my best to sthenise him. His pain was much greater than that in the other cases, so I gave him in addition frequent doses of sedative internally. $\mathbf{A s}$ far as we can judge from results the counter-irritation of the turpentine fomentations was of the greatest service to him.

By some persons a blister is always applied in cases of pleuritic effusion, and with a view, I believe, of removing the tluid by withdrawing it into the blisters which are raised; but if we look at the quantity contained in the chest, and the quantity which the blister "draws," this action is seen to be clearly impossible. The only other way in which it can act is as a counter-irritant, and $I$ think in this way it is inferior either to mustard or turpentine, and has the disadvantage of not being able to be repeated.

With a view of promoting absorption some physicians have adrocated bleeding in cases of pleuritic effusion. There can be no doubt that the abstraction of blood increases the rapidity of absorption, and, therefore, that this practice stands on rational theoretical grounds; but there are strong practical objections to it; it certainly in its general results tends in exactly the opposite direction to that which the rest of our therapeutics is directed to; and in the opinion of some, and among them the late Dr. Todd, has a direct tendency to increase the effusion, by impoverishing the blood and rendering it more watery and prone to passive transudation. I have heard Dr. Todd say myself that he thought, if in any 
given case of pleurisy you wanted to produce effusion, nothing would be so likely to do so as to bleed your patient. At any rate, if we could, by reducing the contents of the blond-vessels, generate an endosmotic current into them, and so favour absorption without impoverishing the blood, it would be vastly better. I am not sure that this might not be done by giving occasional smart doses of hydragogue cathartics; in any future case of hydrothorax, I should feel very much disposed to try it ; carefully keeping up my patient at the same time in every way, by tonic, stimulant, and food, to antagonise the lowering effect of the catharsis.

The only part of my treatment about which I have any doubt is about the value of the iodine. In any case in which one wants to promote absorption, one gives iodine, as a matter of course, externally, internally, and both; and one cannot doubt that locally its effects in this way are sometimes very striking. But if you were to ask me if, in any given case of hydrothorax, I had any tangible and irrefragable proof that the fluid had disappeared the sooner for its administration, I should say I had not.

\section{Cramsattions of 迶ramethes.}

\section{EAST ANGLIAN BRANCH.} PRESIDENT'S ADDRESS.

By Edward Copeman, M.D., Physician to the Norfolk and Norwich Hospital.

[Delivered at Yarmouth, June 26, 1863.]

Gentlemen,- In entering upon the office you have done me the honour to confer upon me (that of President of the Eastern Branch of the Jritish Medical Association), I will not detain you with needless remarks about my own unfitness; but leaving the well beaten track of personal apology, I will enter at once upon what I conceive to be my duty in the position in which you have placed me. Let me, however, in the first place, offer you my sincere thanks for the confi. dence which has led you to entrust me with the conduct of the affairs of this Brunch for the ensuing year, and still more for the friendly feeling which $I$ am fully aware bas influenced you in giving me your valuable support and countenance on the present occasion.

Gentlemen, if we desire to see our noule profession holding the position it so well deserves, occupying a high rank in the social as well as in the moral and scientific world, and enjoying the good opinion and respect of society in generul, we must encourage friendship and even affection amongst ourselves: discourage all animosity and ungenerous opposition; uphold as a body all worthy efforts to increase the respectability and usefulness of our calling, and never on any pretence whatever enter into a combination to oppress the less fortunate, or wound the feelings of the more humble; or, worst of all, increase the burden of a professional brother who may be labouring under some grievous imputation without, perhaps, any just or real grounds. At first sight, we
might think such a remark superfluous, and aimed against an imaginary evil; but is it really so? Have we not seen in several instances how readily some of our body have lent their aid in the prosecution of a professional brother, when there has not been a tittle of reliable evidence against him, and where a prosecution could not have been entered upon with the slightest chance of success had it not been for the help afforded by the opinions of medical men? It is surely but a poor com- pensation to the injured feelings of a sensitive man to be told, when such a trial is over, that he has the sym. pathy of the profession, and that his expenses will be paid by subscription! Gentlemen, he has a right to the sympathy of the profession beforehand, and no medical man ought to allow himself to be placed in a witness-box under such circumstances, without the strongest conviction in his own mind, based upon facts, that the suspected party is really guilty of the charges brought against bim. And even then, if he feel it his duty to give evidence in a court, that evidence should not be founded upon, or guided by, opinion, but solely upon facts and the conclusions to which those facts direct him.

No society, I believe, has ever encouraged friendship and sociability among its members more than that which we are met here today to commemorate; and I cannot help thinking that it is yet fated to be the instrument of very much benefit to our profession. The British Medical Association, formerly the Provincial Association, is coeval with my own professional life, being founded in 1832 , the year when I completed my professional education by passing the College of Surgeons. In 1835, a medical society was formed to embrace the Eastern Counties, of which the late Mr. Cross was the founder and honorary secretary; and the inaugural meeting was held at Bury. But in the following year, this was dissolved as a separate society; and, scon after the fourth anniversary meeting of the Provincial Association at Manchester in 1836, its members joined the Parent Society as the Eastern Branch, setting an example of forming Branches which has since been followed in numerous instances, and even by the metropolis itself. I was a very early member of this Eastern Branch, and find, on referring to the JounNal, that at a meeting held at Bury, in September 1840 , a paper of mine was read " On the impropriety of Blood-letting in certain Apoplectic and Paralytic Seizures." I do not pretend to have been the first to place before the profession the impropriety of indiscriminate bleeding in such cases; but at all events, it was at that time a comparatively new dectrine in this country, and $I$ have lived long enough to see it entertained and adopted almost universally by the profession.

The period which dates from the commencement of this Association has been one of great progress in the medical world. The stethoscope had not been long in use, and was understood only by the few in the early days of this society; but now it is indispensable to the practitioner, and almost answers the purpose of second sight. The chemical examination of the urine was at that time but little practised ; and much of the early success of Dr. Jephson of Leamington was due, I believe, to the point he made of examining his patients' urine, by which he produced a strong impression on their minds that he was adopting a novel and probably beneficial mode of distinguishing the nature of their diseases. What has it come to now? Why, it has become a most important means of diagnosis; it has led to most interesting discoveries as to the nature of various diseases; and, no doubt, has also contributed to the improvement of medical treatment.

'The history of this Association also embraces much of the history of the microscope. When I began practice, it was scarcely ever employed by medical men, but now every student possesses a microscope ; and many practitioners have fuund it an important aid in diagnosis, to say nothing of the interest it las added to the study of the minute anatomy of tissues. Then, as to literature, what a vast amount of valuable information has been placed before us within the same period of time! The first part of Copland's Dictionary was published in 1833, and the learned author has lived to complete that gigantic work of his own hands, and still lives to practise the profession he has so much bonoured by his industry and talent. A Sydenbam Society has lived and died, 